\title{
Simulation of Non-Newtonian Fluids using Modelica
}

\author{
Pooyan Jahangiri $\quad$ Rita Streblow Dirk Müller \\ RWTH Aachen University - E.ON Energy Research Center \\ Mathieustr. 10, 52074 Aachen, Germany \\ pjahangiri@eonerc.rwth-aachen.de
}

\begin{abstract}
Many fluids used today in different applications show a non-Newtonian behavior. In order to simulate this behavior, many different approaches exist but are not fully implemented in a simulation program. One of the problems with these kinds of simulations is the lack of compatibility with existing models. This makes the modeling very time consuming.

In this paper, a simple approach is shown that provides a general set of equations which can then be used to model both Newtonian as well as nonNewtonian behavior of fluids in the same model in Modelica. Since the implementation is in base models, existing components can easily be used to simulate non Newtonian fluids without sacrificing simulation times.
\end{abstract}

Keywords: Non-Newtonian; Medium Model; Pressure Drop

\section{Introduction}

In many applications such as food industries, residential heating and cooling systems, some power plants as well as other energy systems, a nonNewtonian fluid is chosen as the working fluid. The non-Newtonian behavior has a great influence on both flow as well as heat transfer properties of the fluid; therefore, for simulation of such systems, it is necessary to have compatible models and components with this type of fluids.

\section{Theory}

\subsection{The Governing Equations}

Non-Newtonian fluids are fluids in which the viscosity changes with respect to the applied stress. According to the correlation between the shear stress and shear rate, fluids can be divided into different categories (see Figure 1).

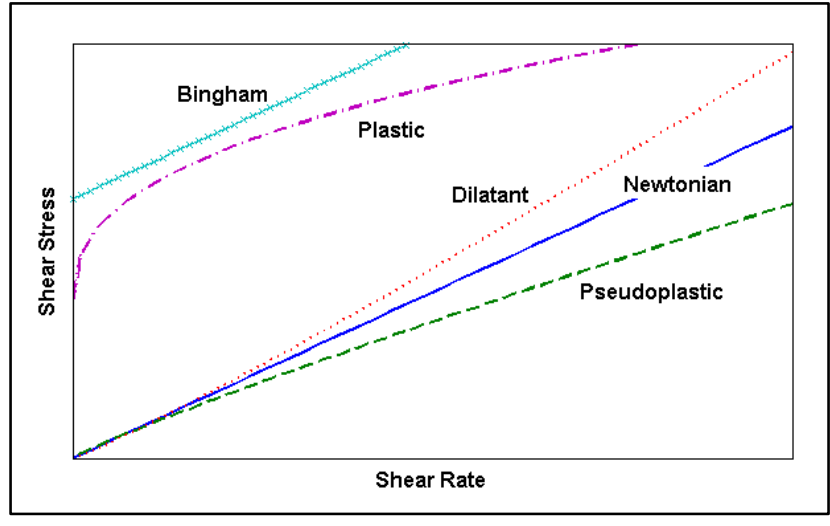

Figure 1: Fluid classification according to shear rate and shear stress

Many available fluids can fully or partly be described by Ostwald-de Waele relationship (Powerlaw fluids) shown in equation (1).

$$
\tau=K \dot{\gamma}^{n}
$$

where

$$
\begin{array}{lll}
\tau & : & \text { Shear stress }[\mathrm{Pa}] \\
K & : & \text { Flow consistency index }\left[\text { Pa. } \mathrm{s}^{\mathrm{n}}\right] \\
\dot{\gamma} & : & \text { Shear rate }\left[\mathrm{s}^{-1}\right] \\
n & : & \text { Flow behavior index }
\end{array}
$$

Flow behavior index " $n$ " as well as flow consistency index " $K$ " are among the properties of the fluid and are considered constant at a given temperature.

By " $n$ " equal 1, the Ostwald-de Waele relationship describes Newtonian fluid behavior. For $n<1$, Pseudoplastic fluids and for $n>1$ Dilatant fluids can be described.

\subsection{Pressure Drop}

For the calculation of the pressure drop in a pipe, when the mass flow rate is known, the dimensionless Darcy friction factor " $\lambda$ " as well as physical parameters of the pipe are used according to equation (2) [1]. 


$$
\Delta p=\lambda \cdot \frac{l}{D} \cdot \frac{\rho \cdot v^{2}}{2}
$$

where

$$
\begin{array}{lll}
\Delta p & : & \text { Pressure drop }[\mathrm{Pa}] \\
\lambda & : & \text { Darcy friction factor } \\
l & : & \text { Length of the pipe }[\mathrm{m}] \\
D & : & \text { Diameter of the pipe }[\mathrm{m}] \\
\rho & : & \text { Density of the fluid }\left[\mathrm{kg} / \mathrm{m}^{3}\right] \\
v & : & \text { Flow velocity }[\mathrm{m} / \mathrm{s}]
\end{array}
$$

In order to calculate the Darcy friction factor, a Fanning friction factor " $f$ " has been introduced by [2] and is shown in equation (3):

$$
f=\frac{\lambda}{4}
$$

The Fanning friction factor for the laminar region can be calculated from equation (4) and for turbulent region from equations (5) and (6) where "Re" corresponds to the Reynolds number. [2, 3, 4]

$$
\begin{gathered}
f=\frac{16}{R e} \\
f=0.0014+\frac{0.125}{R e^{0.32}} \\
f=\frac{0.0791}{\sqrt[4]{R e}}
\end{gathered}
$$

\subsection{Reynolds Number}

In order to calculate the Reynolds number for Powerlaw fluids, [2] also introduces the general Reynolds number in equation (7).

$$
R e=\frac{v^{(2-n)} \cdot D^{n} \cdot \rho}{\gamma}
$$

where

$$
\gamma=K^{\prime} \cdot 8^{n-1}
$$

and

$$
K^{\prime}=K\left(\frac{3 n+1}{4 n}\right)^{n}
$$

Note that using $n=1$, the Reynolds number can be simplified to the Reynolds number in Newtonian fluids as in equation (10).

$$
R e=\frac{v \cdot D \cdot \rho}{\mu}
$$

Depending on the fluid, the turbulent region can start from Reynolds number between 4000 up to 70,000. On the other hand, the boundary Reynolds number between the laminar and the transitional region can be calculated according to [5] using equation (11).

$$
R e_{l a m}=\frac{6464 n}{(3 n+1)^{2}} \cdot(2+n)^{\left(\frac{2+n}{1+n}\right)}
$$

\section{Implementation in Modelica}

\subsection{Existing Flow Models}

In Newtonian fluids, the viscosity does not depend on the applied stress or in other words the volume flow rate; hence, it can be calculated within the medium model using just the base properties of the fluid such as the pressure and the temperature.

In existing models in Modelica standard library, at each calculation step, the viscosity of the fluid is calculated within the medium model. This is then used to estimate the Reynolds number according to equation (10). By knowing the Reynolds number, the flow region can be chosen and the governing equations for that region are used to calculate the Darcy friction factor and then the pressure drop.

Note that since Reynolds number is a function of velocity, the procedure mentioned above is only valid when the velocity is known by knowing either the mass flow rate or the volume flow rate and the geometry of the pipe. For this reason, a new set of equations are also implemented to calculate the mass flow rate in a pipe when the pressure difference between two pints is the known variable.

This is helpful for many hydraulic components such as pumps which produce a certain pressure difference and the result will be the flow of the medium; therefore, in order to be able to simulate the flow of the medium properly, it is also necessary to be able to calculate the mass flow rate from the pressure drop.

In this procedure, a second friction factor " $\lambda_{2}$ " is introduced which is independent of the velocity and is shown in equation (12).

$$
\lambda_{2}=\frac{2 \cdot D^{3} \cdot \rho}{l \cdot \mu} \cdot \Delta p
$$

Using the second friction factor, the Reynolds number can be estimated by the Reynolds number equation for the laminar region and be corrected if the estimation result falls above the turbulent boundary 
using the equations governing the turbulent region. The velocity and the mass flow rate are then calculated using the Reynolds equation.

\subsection{Non-Newtonian Medium Model}

Since the viscosity of Non-Newtonian fluids cannot be calculated using only the base properties, the main calculation should be in the flow model. In order to correlate flow model and medium model with the smallest change possible, an extra function is required in the "Partial Medium Model". This function will describe the flow behavior index " $n$ " and is written as follow:

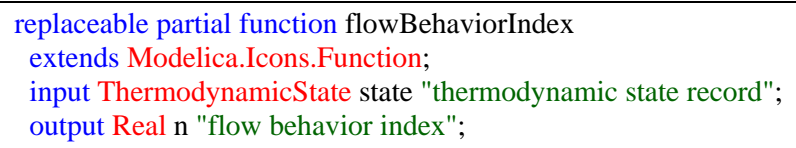

Since the flow behavior index is only a function of the states of the fluid such as the temperature, it can be defined and calculated in the medium model. By adding this partial function for the definition of the flow behavior index in the base medium model, implementing the governing equations or tables for all the fluid models is made possible.

In case the fluid is a Newtonian fluid such as water, the flow behavior index should be set to the constant number of " 1 ".

By comparing equations (7), (8) and (10) it can be seen that having $\mathrm{n}=1$, the coefficient $K^{\prime}$ is equal the dynamic viscosity " $\mu$ ". This means that the "dynamicViscosity" function in the partial medium model can also be used to calculate the consistency dex $K^{\prime}$. Like the flow behavior index, the consistency index does only depend on the base properties of the fluid and not the flow parameters. Therefore it can also be calculated in the medium model.

\subsection{Non-Newtonian Flow Models}

Having the flow behavior and consistency indices from the medium model, the Reynolds number can be calculated when the volume flow rate is known using the general Reynolds number shown in equation (7). Using the Reynolds number, the fanning friction factor can be calculated using equation (4) for the laminar region and equation (6) for the turbulent region. The pressure drop is then calculated with the help of equations (2) and (3).
For the transitional region between laminar and turbulent, the laminar region is connected to the turbulent region using a cubic Hermite spline.

As already discussed in the existing flow models, it is necessary to have a function for calculation of mass flow with respect to pressure difference in the system. Since pressure drop as well as Reynolds number and hence the Darcy friction factor are a function of velocity which is derived from the mass flow rate, here is also not possible to use the general Reynolds number and Darcy friction factor directly for these calculations. To solve the problem, a variable which is independent of velocity is introduced in equation (13) and is called the modified Darcy friction factor " $\lambda_{m}$ ".

$$
\lambda_{m}=\lambda \cdot R e^{\left(\frac{2}{2-n}\right)}
$$

Combining equations (2), (7) and (13), the modified Darcy friction factor can be calculated as follow:

$$
\lambda_{m}=\frac{2 \cdot D^{\left(\frac{2+n}{2-n}\right)} \cdot \rho^{\left(\frac{n}{2-n}\right)}}{l \cdot \gamma^{\left(\frac{2}{2-n}\right)}} \cdot \Delta p
$$

By having a flow behavior index of "1" as for Newtonian fluids, the modified Darcy friction factor is reduced to equation (12).

When the modified Darcy friction factor is known, the Reynolds number can be calculated under the assumption that the flow is laminar using equation (15) achieved from equations (3), (4) and (13).

$$
R e=\left(\frac{\lambda_{m}}{64}\right)^{\left(\frac{2-n}{n}\right)}
$$

If the calculated Reynolds number according to equation (15) is greater than the turbulent Reynolds number, then the Reynolds number is calculated for the turbulent region using equation (16) derived from equations (3), (6) and (13).

$$
R e=\left(\frac{0.3164}{\lambda_{m}}\right)^{4\left(\frac{n-2}{n+6}\right)}
$$

By knowing the Reynolds number, the mass flow rate can be calculated as follow: 


$$
\dot{m}=\left(\frac{\left(\frac{\pi}{4}\right)^{2-n} \cdot \gamma \cdot R e}{D^{3 n-4} \cdot \rho^{n-1}}\right)^{\frac{1}{2-n}}
$$

The transitional region here is also generated by a cubic Hermite spline as before.

\section{Simulation Results}

The specified functions are directly implemented in the "detailed wall friction model". The model is tested for a Paraffin-Water dispersion shown in Figure 2 with $30 \%$ paraffin dispersed in water. The fluid shows a pseudoplastic behavior. The dispersed paraffin goes through a phase change at a certain temperature which not only affects the thermal properties but also the flow properties of the fluid.

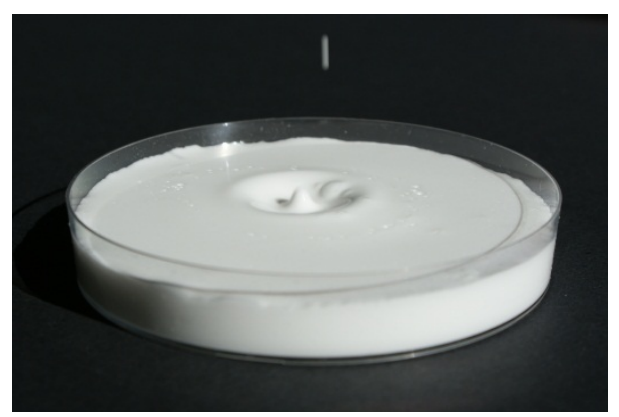

Figure 2: Paraffin-Water dispersion used as working fluid in energy systems

The measured flow behavior and consistency indices at different temperatures for the Paraffin-Water dispersion are shown in Figure 3 and are implemented in the medium model.

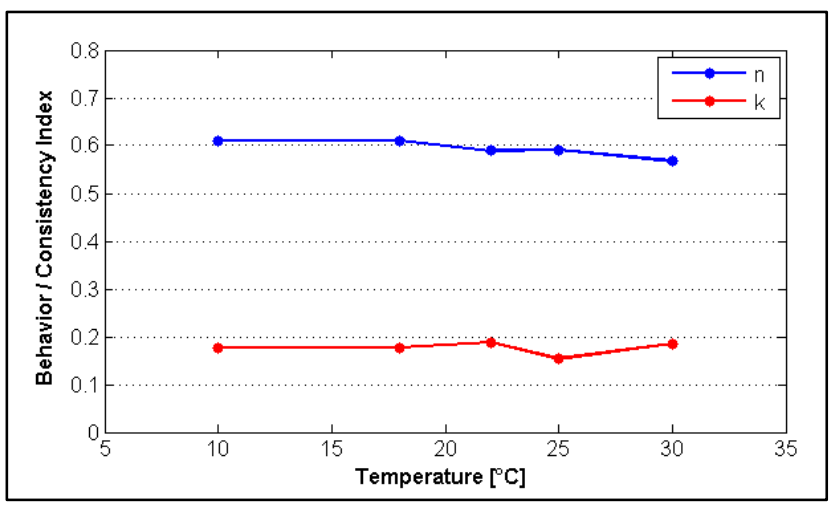

Figure 3: Measured flow behavior and consistency indices for Paraffin-Water dispersion ${ }^{1}$
To calculate the pressure drop from a known mass flow rate, Paraffin-Water dispersion model at $22^{\circ} \mathrm{C}$, with $\mathrm{n}=0.5889$ and $\mathrm{K}=0.1877$, is used in a simple simulation model (consisting of a pipe with a length of $1 \mathrm{~m}$ and diameter of $0.05 \mathrm{~m}$. The results are shown in Figure 4.

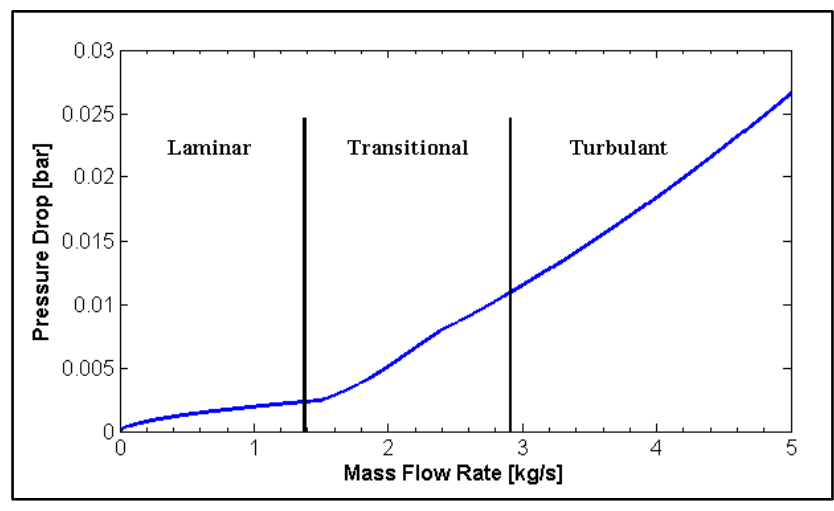

Figure 4: Pressure drop of Paraffin-Water dispersion with respect to mass flow rate

The mass flow rate is then calculated in a simple simulation model when the pressure difference is known for a pipe with $1 \mathrm{~m}$ length and $0.05 \mathrm{~m}$ diameter and is shown in Figure 5.

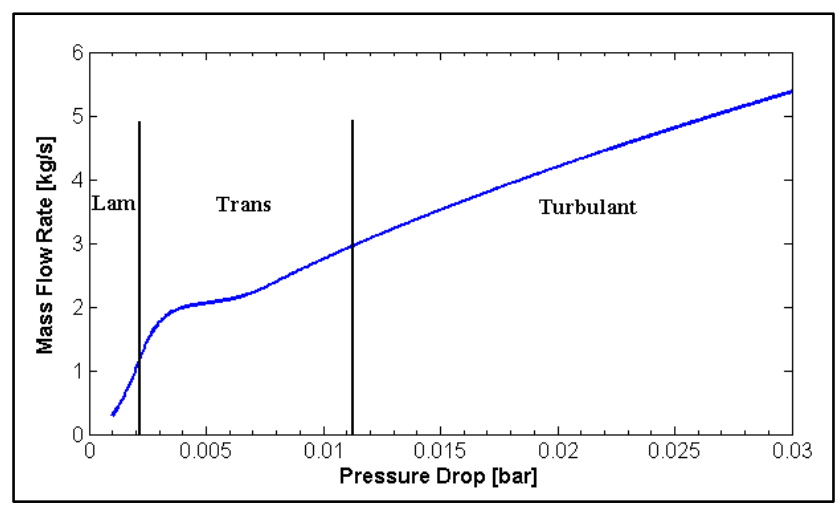

Figure 5: Mass flow rate of Paraffin-Water dispersion with respect to pressure drop

Since the model can be used for all the fluids governed by Ostwald-de Waele relationship, the pressure drop is compared for simulations with 3 different flow behavior indices and is shown in Figure 6 . The closer the behavior index to 1 is, the closer the fluid behaves as a Newtonian fluid.

\footnotetext{
${ }^{1}$ Data provided by Fraunhofer UMSICHT
} 


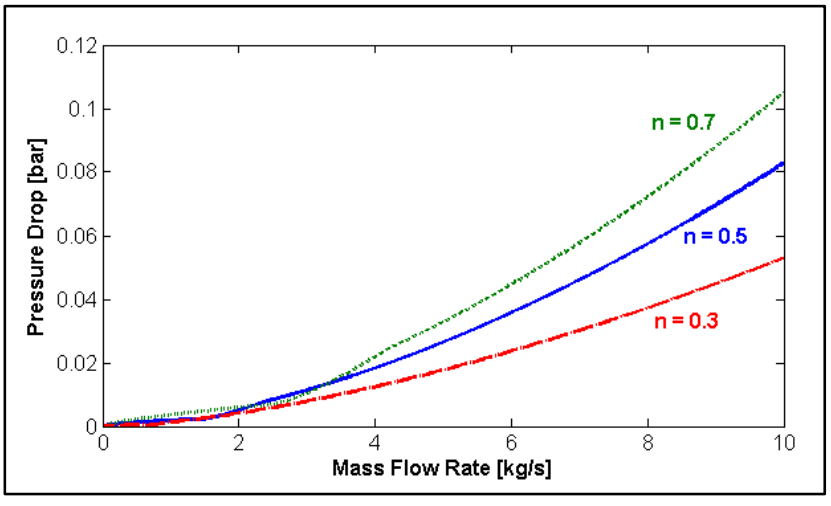

Figure 6: Comparison between different pressuredrops in fluids with different flow behavior indices

\subsection{Compatibility}

In all the equations described for the non-Newtonian flow, the equations for the Newtonian fluids are derived when the flow behavior index is set to " 1 ", These are the exact equations which are already implemented in the wall friction model in Modelica standard library. Therefore, the same flow model can be used for all the existing medium models in all existing components without any compatibility issues.

Since the non-Newtonian flow model is an extension to the available flow model in Modelica 3.2, it is also compatible with the entire fluid library. This will omit the need to design new components just for non-Newtonian systems.

\subsection{Simulation Times}

To substitute the existing model for the general flow model, it is important to maintain the fast simulation speed. Therefore, a simple dynamic simulation is done using Paraffin-Water dispersion and water with the general model described in this paper and is compared to the same simulation setup using water and the existing flow models. The CPU times are compared in Table 1.

Table 1: CPU Time comparison between different models and fluids

\begin{tabular}{|cc|c|}
\hline \multicolumn{3}{|c|}{ CPU Time } \\
\hline \multicolumn{2}{|c|}{ Modified Model } & Existing Model \\
\hline Dispersion & Water & Water \\
\hline $0.106 \mathrm{~s}$ & $0.109 \mathrm{~s}$ & $0.105 \mathrm{~s}$ \\
\hline
\end{tabular}

It can be seen that although more complicated equations are used, the solving time stays almost in the same range. This will result in simulation times which are almost the same as in existing flow models in Modelica.

\section{Conclusions}

There are many applications for simulating the behavior of non-Newtonian fluids such as food processing plants and energy distribution systems.

In order to implement the non-Newtonian behavior, an extra function is added to the base medium model. This function describes the flow behavior index of a fluid and enables the interaction between the medium model and the flow model. The flow behavior index corresponds to the degree of non-Newtonian behavior of each fluid. Having the necessary interaction between the models, more general equations regarding pressure drops in the system can be implemented. These equations contain both the Newtonian as well as non-Newtonian behavior of a fluid.

Since the changes are in the base models, any other component that uses the model directly or indirectly can be used for the simulation of both Newtonian and non-Newtonian fluids without any additional changes and compatibility issues.

Using the flow behavior index, the non-Newtonian behavior of fluids can later be expanded to the heat transfer properties of fluids in the Modelica thermal libraries.

\section{Acknowledgement}

Grateful acknowledgement is made for the financial support by BMWi (Federal Ministry of Economics and Technology), promotional reference 032747B and for great help and support by Fraunhofer UMSICHT. 


\section{References}

[1] Wagner, W.; Strömung und Druckverlust; 3rd Ed. Germany : Vogel Publication, 1992.

[2] Metzner, A.B. und Reed, J.C.; Flow of NonNewtonian Fluids - Correlation of the Laminar, Transition, and Turbulent-flow Regions; A.I.Ch.E. Journal. December 1955, pp. 434440.

[3] Dodge, D.W. und Metzner, A.B.; Turbulent Flow of Non-Newtonian Systems; A.I.Ch.E. Journal. June 1959, pp. 189-204.

[4] Wronski, Jorrit.; Untersuchungen zur Bestimmung des Feststoffgehalts von Dispersionen - Entwicklung eines Ladesensors für PCS-Systeme; Ruhr-Universität Bochum Lehrstuhl für Feststoffverfahrenstechnik, 2010.

[5] Chhabra, R.P. und Richardson, J.F; NonNewtonian Flow in the Process Industries; Oxford, 1999. 